Revue de géographie historique

$14-15$ | 2019

Impacts environnementaux et approches spatiales de la Grande Guerre

\title{
L'archéologie champenoise et le Premier Conflit mondial, géologie et recherche
}

Jean-Jacques Charpy

\section{OpenEdition}

\section{Journals}

Édition électronique

URL : https://journals.openedition.org/geohist/590

ISSN : 2264-2617

\section{Éditeur}

Association française de la Revue de géographie historique

Référence électronique

Jean-Jacques Charpy, "L'archéologie champenoise et le Premier Conflit mondial, géologie et recherche », Revue de géographie historique [En ligne], 14-15 | 2019, mis en ligne le 28 mai 2018, consulté le 12 juin 2021. URL : http://journals.openedition.org/geohist/590

Ce document a été généré automatiquement le 12 juin 2021.

\section{(c) (i) (2)}

Ce(tte) œuvre est mise à disposition selon les termes de la Licence Creative Commons Attribution Pas d'Utilisation Commerciale - Pas de Modification 4.0 International. 


\section{L'archéologie champenoise et le Premier Conflit mondial, géologie et recherche}

Jean-Jacques Charpy

\section{Introduction}

1 Les archéologues de la seconde moitié du XIX siècle n'ont accordé à la géologie, dans leurs travaux, qu'une attention très limitée. On peut considérer que cette science ne les intéresse très directement que pour le domaine environnemental de leurs découvertes. On trouve donc au mieux des considérations liées à l'oro-hydrographie et au substrat, qui dans la très grande majorité des cas, est constitué de craie franche ou de sa forme modifiée par la cryoturbation appelée soit graveluche, soit tuffeau. La plupart des pionniers ont débuté leurs recherches sur le terroir de leur commune. Ils n'évoquent donc pas le milieu crayeux qui pour eux est d'une évidence première. Lorsqu'ils sortent de cet espace, ils s'attachent à chercher là où l'on procède à des travaux d'aménagement du territoire (Charpy, 2010). Les atlas cantonaux leur fournissent une aide secondaire lorsqu'il y figurent les carrières, terrières, crayères et autres exploitations d'argile (Poinsignon, 1877). Ces excavations permettent de déceler les anomalies et les surcreusements dans les fronts de taille.

2 Un point marquant a très longtemps hanté l'esprit des archéologues; c'est celui du remplissage en terre noire des fosses. On citera en premier lieu C. Bosteaux-Paris: «[...] Les corps déposés avaient été recouverts d'une terre noire très fine, comme une poussière de charbon; cette terre noire était la cendre du foyer de la hutte du défunt, car tous les foyers sont remplis de cette cendre [...]." (Bosteaux-Paris, 1883). Ce type de constat s'est multiplié jusque pendant la Grande Guerre: «au-dessus de la ferme des Marquises [Prunay, Marne], dans un secteur de première ligne, un intéressant cimetière dont les tombes à terreau noir, coupées transversalement, se distinguaient nettement sur les parois blanches des boyaux» (Olivier, 1916). 
3 En 1971, on pouvait encore lire: «[...] certains ont pensé que cette terre noire avait été extraite du foyer de l'habitation du mort (...)il ne peut guère s'agir de terre noire issue essentiellement d'un terrain sacré (...)il n'est pas possible non plus que ces mètres cubes de terre noire proviennent exclusivement de la seule décomposition des feuillages, fleurs ou mottes de gazon jetés dans la tombe (...). On peut donc conclure que cette terre noire provient de la décomposition de l'inhumé, de ses parures et offrandes funéraires, mais aussi d'une importante quantité de débris d'origine végétale » (Bretz-Mahler, 1971). La réponse a été apportée en septembre 2005 à Bernard Lambot avec l'étude d'un prélèvement du comblement de la tombe à char de Prunay «La Voie de Baconnes" (Marne) par le Laboratoire départemental d'analyses et de recherche de la Station agronomique de Laon (Aisne) : il s'agit d'humus. Lorsque le baron Joseph de Baye (de Baye, 1881) aborde la stratigraphie géologique du site des «Pâtis » de Fèrebrianges (Marne), il note que l'humus représente une épaisseur de $30 \mathrm{~cm}$ environ, que le lœss ou limon celle d'un mètre puis que les sables tertiaires avec grès souvent dénudé, celle de 2 mètres et plus. Si l'attribution est juste pour les deux premières, elle est imprécise pour la dernière puisqu'il n'évoque pas le Stampien. L'exemple qui suit est choisi pour sa situation géographique, à l'est du département et sur une petite terrasse alluviale. On extrait la citation ci-dessous de la publication de la nécropole de «Charvais » (Mougin, 1877) à Heiltz-L'Evêque (Marne). On cite: «[...] c'était d'abord le seul endroit qui, lors des débordements de la Chée, restât praticable. Une légère élévation l'empêchait d'être couvert par les eaux (...) je savais que depuis longtemps les tireurs de grève (...) trouvaient parfois des squelettes. ». On ne peut être plus lapidaire pour définir l'environnement géologique de cette nécropole. Pour évoquer le milieu non crayeux de la Marne, l'enceinte d' « Haulzy » à Vienne-la-Ville (Goury, 1911) illustre un cas non éloigné des précédents. Ainsi lit-on : «[...]au confluent de la Tourbe et de l'Aisne, au nord-ouest du village (...) s'étend un plateau triangulaire: de la vallée de la Tourbe, il s'élève en pente douce pour tomber à pic sur l'Aisne; au sud des pitons crayeux limitent la base du triangle. Ce plateau est constitué par la gaize, roche grise, argilo-siliceuse, qui présente en surface un terrain léger et sableux ». Dans ce cas, la description est un peu enrichie.

4 La perception de la géologie change considérablement lorsque l'on aborde le néolithique avec les haches polies en roches rares : jadéite, chloromélanite, etc. (Baye, 1886) ou bien lorsqu'il est question de la parure. Pour ces dernières, on cherche à identifier les espèces fossiles qui la composent. En ce qui concerne la phase ancienne de cette période, on peut se rapporter à la tombe de Frignicourt (Marne) et l'on cite (Mougin, 1899) : " [...] une coquille en cardium, percée d'un trou à son extrémité (...) une petite plaque en matière schisteuse percée de deux trous et une pendeloque percée d'un trou taillée dans une coquille d'unio.». Quelques années auparavant dans sa monographie, traitant de ce que l'on sait être aujourd'hui le néolithique récent et final, Joseph de Baye (Baye, 1880), identifie tous les tests des mollusques et gastéropodes trouvés dans les hypogées des marais de Saint-Gond. Les espèces sont nombreuses et présentes dans le Lutétien mais, au XIX ${ }^{\mathrm{e}}$ siècle prime la reconnaissance. C'est ainsi que l'on peut citer d'après cet auteur et sachant que bien des identifications ont changé : les orbitolines (d'après Parker et Jones), les dentales (Fig. 1), les cardes, les vénéricardes. Depuis, l'inventaire s'est considérablement enrichi (Polloni, 2007) grâce aux nouvelles découvertes qui se sont succédées jusque ces dernières années. 
Document 1 : Perles en test de dentales. Aulnay-aux-Planches «Au-Dessus du Chemin des Bretons", sépulture collective du néolithique récent (cl. J.-J. Charpy)

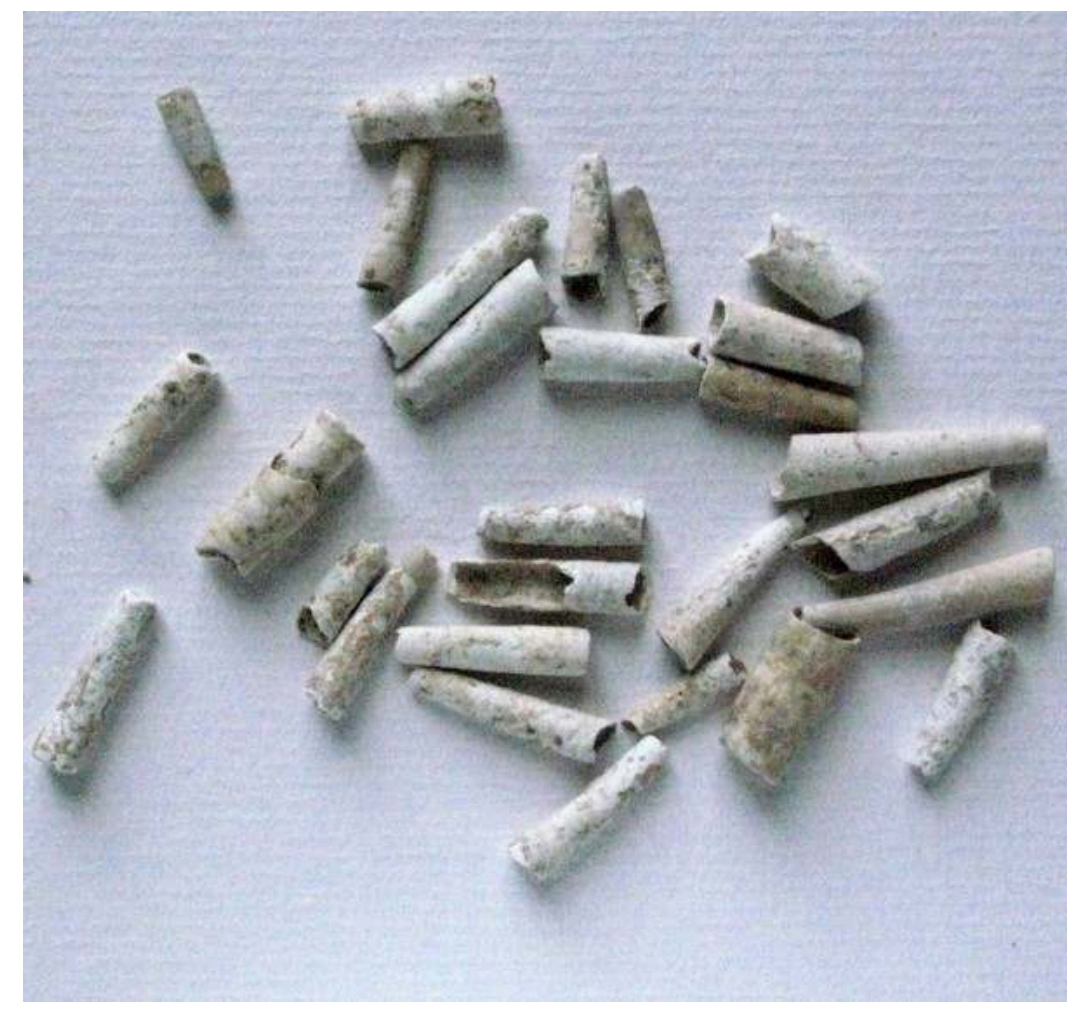

5 Si le constat que l'on vient de faire ne paraît pas extrêmement brillant pour les archéologues champenois, un regard porté chez nos proches voisins de l'est montre qu'ils ont cherché l'aide de l'université de Nancy et de la géologie pour expliquer, dès la décennie 1870 (Bleicher, 1880), la vitrification et la datation du rempart du massif du Hartmannschwillerkopf (Vieil Armand depuis la première Guerre Mondiale) près de Wattwiller (Haut-Rhin). Et c'est dans le prolongement de la situation que l'on vient de décrire que survient le conflit de 1914-1918 et un médecin d'écrire : «La masse énorme de terre remuée tout le long des lignes méritera après la guerre une étude attentive. Les géologues y trouveront sans bourse délier, le plus admirable choix de coupes superficielles de terrain qui se soit jamais vu. Et les archéologues feront sans aucun doute en circulant dans les boyaux d'inattendues trouvailles. » (Olivier, 1916).

\section{L'archéologie en Champagne pendant le Premier Conflit mondial}

6 La recherche allemande fréquentait et connaissait la Champagne bien avant la Grande Guerre. Certains notables payaient même des archéologues pour enrichir leurs collections et celles des musées. On peut citer le cas du baron Johannes von Diergardt (1859-1934) et ses rapports avec Jean-Baptiste Bénoni Lelaurain (1850-1905). La connaissance du milieu local par les services de l'Armée allemande permet d'expliquer la disparition d'un certain nombre de collections privées (Louis Simonnet, 1874-1941, à Hauviné, Ardennes) mais ce sont des vols partiels de rapine, pour l'année 1914, qu'ont connu certaines autres : les collections russes et caucasiennes de Joseph de Baye et celle d'archéologie locale d'Augustin Roland (1867-1943). Mais plus importantes encore 
furent les destructions d'ensembles constitués patiemment que des bombardements ont anéantis : celui de Jules Dupuis (1866-1949), déménagé à la hâte de Pontfaverger à Sommepy (Marne), celui d'Henri Gillet (1890-1947), à Reims, jusque ceux installés dans le Palais du Tau et disparus sous les bombes incendiaires du 19 septembre 1914.

7 C'est ensuite en 1917 que disparaissent les salles du musée de l'hôtel de ville de Reims. A la suite de ces désastres, H. Jadart (1847-1921) écrivait à Héron de Villefosse (1845-1919) : "L'armée de nos ennemis, continuant son ceuvre, a détruit les précieux documents que, depuis près d'un siècle, Duquenelle, Fouché, Habert, Favre, Bosteaux et d'autres avaient recueilli avec un soin pieux; les registres d'inventaire et les catalogues manuscrits ont été la proie des flammes. L'installation de ce beau Musée était à peine terminée quand la guerre éclata ; il a disparu avant même d'avoir été inauguré ; à côté de tant d'autres, c'est un désastre irréparable pour la Champagne, désastre dont tous les archéologues éprouveront un profond regret; il n'en reste rien.» (Jadart, 1914). On sait par un témoignage oral, recueilli par Pierre Roualet (1930-2015), que les archéologues, Marie-Louise Morgen (1895-1983) et son père Maurice Bry (1872-1958), ont fouillé dans les cendres du musée pour tenter d'y recueillir et sauver quelques objets. On peut relater un autre épisode particulier et lié à la première bataille de la Marne des 5 et 6 septembre 1914. Les notes d'Augustin Roland lévouent ainsi qu'une partie de la population de Villevenard est allée se cacher dans les hypogées néolithiques des "Ronces». Si les carnets ont été perdus dans les bombardements de juin 1940, cette partie a très vite été publiée (Anonyme, 1915a; Le Goffic, 1916 et 1921). Des photographies (Villevenard) et des graffitis (Vert-la-Gravelle) témoignent de la visite de ces monuments néolithiques par des soldats pendant la durée de la guerre. Pour enrichir la question de la recherche en temps de guerre, on peut aussi signaler la lettre de Léon Bérard (1883-1918) dont on ne connaît pas le destinataire (Bérard, 1920). Elle est datée du 13 septembre 1915 dont on cite l'extrait suivant : "Je suis allé voir si je trouverais chez lui (à Villevenard), à une douzaine de kilomètres d'ici [Bérard à ce moment est à Sézanne], M. Roland, un instituteur fouilleur que je connaissais et avec qui j'avais échangé des pots autrefois. Sa collection n'a pas trop souffert, la maison n'ayant reçu aucun obus, et les Boches n'ont emporté que quelques bijoux mérovingiens, parce qu'ils étaient en or ou en argent. Quant aux grottes néolithiques des environs, elles ont servi de refuge aux habitants pendant la bataille de la Marne. On a même fouillé partiellement, depuis, les débris d'une nouvelle grotte ouverte par un obus allemand [...] ».

8 Augustin Roland (Roland, 1923) aura dû attendre le 8 mars 1915 avant d'être autorisé par l'autorité militaire à accéder au site que chaque jour, il pouvait voir ou presque sur la rive opposée des marais de Saint-Gond depuis son domicile. Cette difficulté à accéder aux tranchées pendant l'année 1915 est parfaitement illustrée par l'aventure de M. Huber qui a passé trois heures au poste de police, accusé d'espionnage, pour s'être rendu dans les tranchées de Chelles (Seine-et-Marne) proches des ballastières (Huber, 1915) tout comme par les propos de Philippe Victor Reynier (1846-1920) relatifs à la recherche paléontologique et archéologique dans le secteur, non éloigné, de Lizy-surOurcq (Seine-et-Marne) : «Il est bon de visiter tous les terrassements. - Si l'on veut éviter toute discussion avec la police, il faut se munir de bons papiers, lors même que l'on serait de la localité où sont creusées les tranchées!». Et plus loin d'ajouter: "L'époque la plus intéressante pour les recherches sera l'époque du comblement des tranchées, car le creusement s'est fait par couche de terrain de $0 m 60$ à $0 m 80$ d'épaisseur; de cette manière il résulte que la partie où l'on pourrait trouver des objets néolithiques se trouve recouverte par des terrains, souvent non remaniés. Les époques acheuléennes et moustériennes peuvent se trouver dans les 
terrains non remaniés. " (Reynier, 1915). Le conseil valait mais ne semble pas avoir pu être suivi dans la zone du front, l'urgence agricole ayant fait nécessité.

Dans un ouvrage récent, on a évoqué quelques-unes des rares fouilles effectuées par l'armée dans la Marne et publiées presque aussitôt (Charpy, 2017). La première est celle de Cauroy-lès-Hermonville près du boyau d'Esternay. On doit la recherche au SousLieutenant Antoine de Traverse (1891- ?) accompagné du Lieutenant Emile Garandeau (1886-1918). Mais c'est à Pierre Gautier, Sous-Lieutenant au 216 R.I. (1884-1917), archiviste de la Haute-Marne, que l'on doit la publication dans le Bulletin archéologiquede 1916. On sait que ce régiment était affecté de février à mai 1916 au secteur de La Neuville et de la Maison bleue entre la ferme du Godat, proche du boyau d'Esternay (Fig. 2), et Sapigneul (village détruit situé entre Cormicy (Marne) et Berry-au-Bac (Aisne)). La découverte est sans doute à placer à la période finale de l'installation du régiment mais plus probablement au début de l'été et l'article écrit à la fin de cette saison, puisque Antoine de Traverse y est mentionné comme l'inventeur et qu'il n'a été nommé au grade de sous-lieutenant que le 30 juillet 1916, et que Garandeau est affecté au $216^{\mathrm{e}}$ R.I. le 30 juillet (Blog d'RV, 2005). On est aussi en mesure d'apporter des précisions supplémentaires sur la découverte (Charpy, 2017) d'une tombe galloromaine (Chew, 1993) à Sarry (Marne) par le Colonel Gustave d'Hannezo (1857-1922). On sait que ce militaire de carrière, rappelé sous le drapeau en 1914, a effectué en Tunisie où il a été affecté, des recherches archéologiques intensives de 1886 à 1904 (Laporte, 2017). Le 19 juillet 1916, on lui confie le commandement du 82 R.I. En septembre, il met au jour la sépulture et le 13 novembre, il envoie les dessins au Comité des travaux historiques et scientifiques dont Salomon Reinach est le responsable pour l'archéologie. Ce dernier a publié la découverte dans la foulée (Reinach, 1917), en commettant une grossière erreur sur la nature de la céramique conduisant à mal dater cette tombe. L'article était-il écrit avant l'arrivée des objets au MAN en décembre $1916 ?$

Document 2 : Localisation du boyau d'Esternay sur le fond de carte militaire.

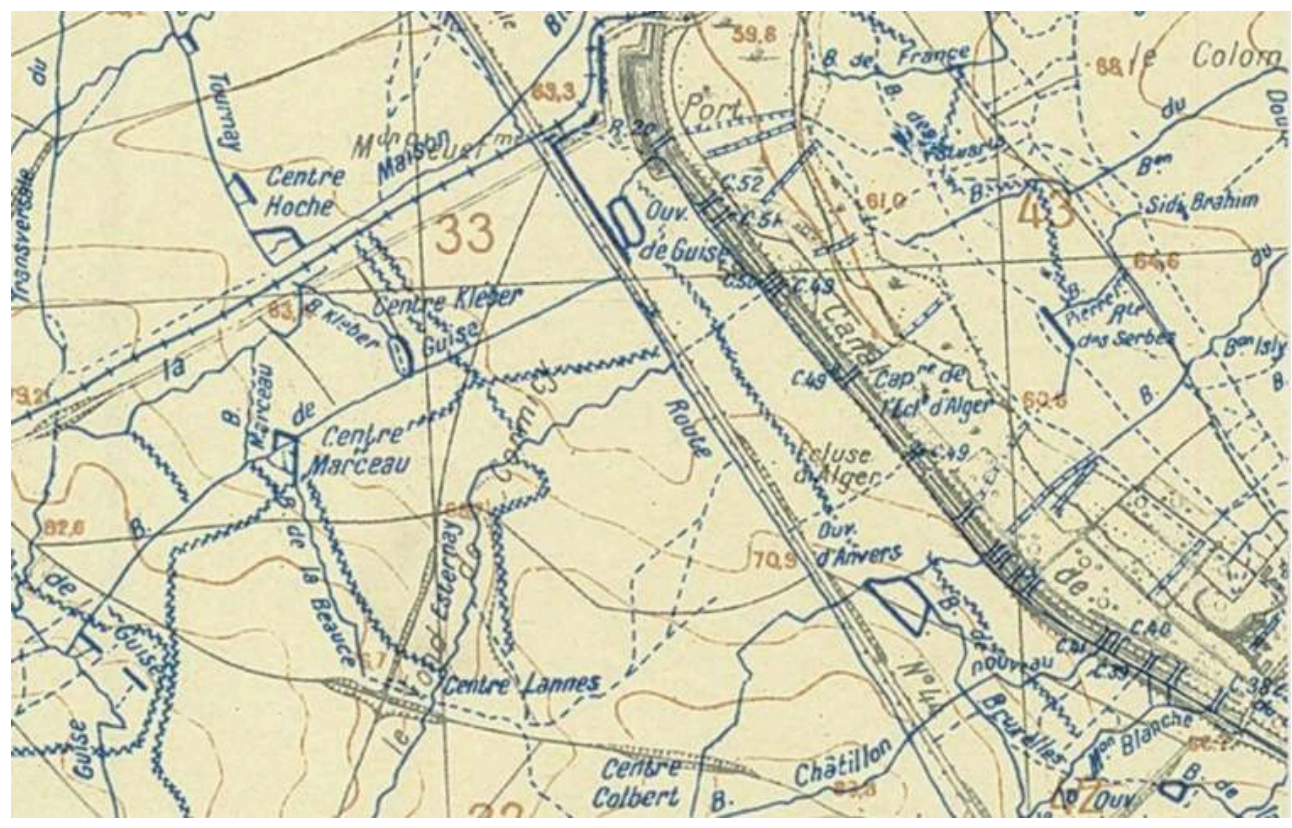


10 Une autre mention d'une découverte archéologique, côté français en Champagne, est celle de la carrière proche de l'arbre signal du schéma directeur du 16 septembre 1918. On sait que la découverte est due au Capitaine Georges Bonneau (1879-1969) du $3^{\mathrm{e}}$ R.A.C. (Crid1418, 2011), dont les archives ont été détruites à l'exception des lettres échangées avec sa femme. On sait que son père était un médecin connu de Toulouse. Il aura donc communiqué ses découvertes à son père, qui les a fait paraître dans le Bulletin de la Société archéologique du Midi de la France de 1914, sous la signature de son fils. On s'était interrogé sur les raisons de la parution de ces trois articles concernant les découvertes. Les recherches menées montrent que pour chacun des cas exposés, il y a une relation avec le milieu historique ou archéologique. Celle de Tahure précède la potentielle circulaire du Comité archéologique des travaux historiques et scientifiques de 1915, suscitée par la Société archéologique de Provence, qui demandait qu'à l'occasion des travaux de tranchées, les découvertes archéologiques soient signalées au Comité. On peut donc témoigner que par deux fois, par Cauroy-les-Hermonville et Sarry, la demande a été observée; mais on vient de montrer que cela a été réalisé par des militaires déjà concernés par ce sujet.

11 Le résultat semble assez pauvre si l'on en croît les entrées au Musée des Antiquités nationales pendant le conflit (Olivier et al., 2015) et les mentions signalées dans le bulletin de la Société préhistorique françaisede 1915 et de 1916, voire dans la presse (Anonyme, 1915b). On doit encore souligner, pour illustrer le sujet, les diverses interventions du Médecin major Jean Emmanuel Paul Trassagnac (1873-1944) qui, vers mars 1915, alors qu'il est en Champagne, nous délivre le contexte général de ses premières découvertes : "[...] la guerre a naturellement tourné depuis bientôt neuf mois mes occupations et préoccupations vers un tout autre objet que la Préhistoire; mais depuis que les tranchées ont commencé à être creusées sur la ligne de feu, tout en m'occupant de mes blessés, je ne négligeais de jeter un regard interrogateur sur la coupe des terrains mis au jour. Ces derniers temps même, profitant d'une accalmie momentanée des combats auxquels mon régiment a pris part, j'ai pu entreprendre des fouilles (Trassagnac, 1915a). Etant donné la proximité des lignes allemandes, éloignées seulement de quelques centaines de mètres du boyau où j'ai surtout opéré ces recherches, et en raison du grave inconvénient qu'il y aurait pour le moment à livrer à la publicité des renseignements qui pourraient être utiles à l'ennemi, il m'est impossible de donner maintenant des précisions topographiques. Après la guerre je fournirai à la Société Préhistorique Française tous les éléments nécessaires (...). Je donne ci-contre un plan des boyaux avec les indications de chaque point fouillé. Je n'ai pu étendre mes recherches dans les terres voisines, à cause du danger d'être aussitôt repéré et canonné par l'artillerie allemande. » (Trassagnac, 1915b) (Fig. 3a, b, c).

12 Comme on pouvait s'en douter, la main ne peut venir rechercher ce que l'œil a perçu que dans les périodes calmes et dans des conditions qui restent bien précaires. C'est ainsi que ce médecin a exploré, en quatre points différents, une fosse de La Tène ancienne, six autres de La Tène finale et de la période gallo-romaine, et signale deux incinérations dont l'une fouillée par lui après communication de l'autre explorée partiellement par le docteur Cousyn (? - ?) à l'entrée du boyau Vauban. Grâce au plan communiqué, on peut restituer assez aisément les lieux des découvertes sur le long du cours de la Suippe à Aubérive et à Saint-Hilaire-au-Temple (Marne) (Fig. 4a, b). Ces informations recoupent pour une part des découvertes faites par des pionniers du XIX siècle (Chossenot, 2004). En 1917, Trassagnac fait don au Musée de la Société préhistorique française $\mathrm{du}$ mobilier trouvé lors de ces premières recherches 
(Trassagnac, 1916). L'année suivante après d'âpres combats, il est mis au repos un temps à Epernay sur l'arrière du front. Il envoie alors des informations à la même revue concernant la découverte de puits funéraires gallo-romains à Tours-sur-Marne «La Noue Morizet" (Marne) qu'il date du Bas-Empire en bon numismate qu'il est (Trassagnac, 1917a), et signe un article après avoir prospecté le plateau et l'enceinte du «Bois des Châtaigniers » à Cerseuil (Marne) commune de Mareuil-le-Port (Trassagnac, 1917a). On peut reconnaître à ce médecin major le titre d'avoir été le plus prolixe pendant le temps de guerre, mais il assouvissait une passion bien antérieure au conflit.

Document 3a : Carte des découvertes publiée par Jean Emmanuel Trassagnac (BSPF, 1915, p. 332).

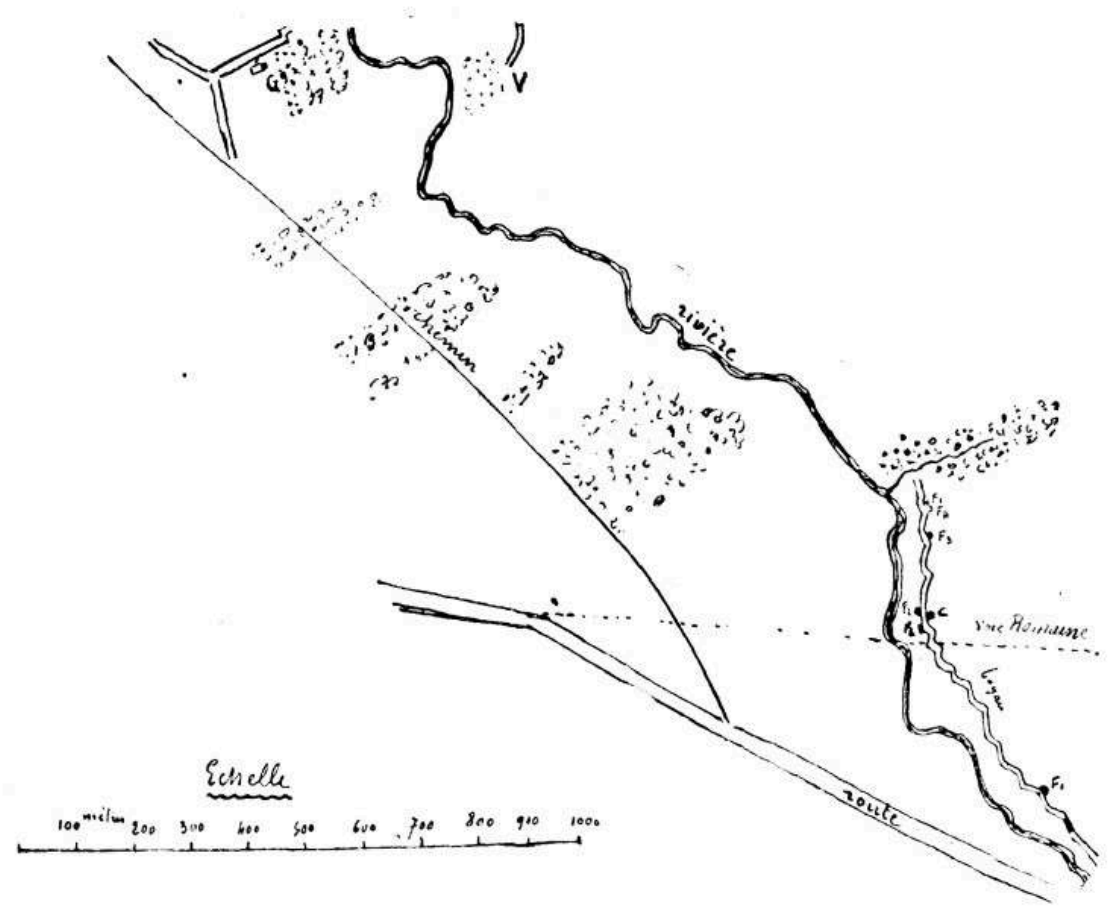


Document 3b : Le même secteur extrait de la carte militaire de Sainte-Marie-à-Py (Marne) du 31 janvier 1918 .

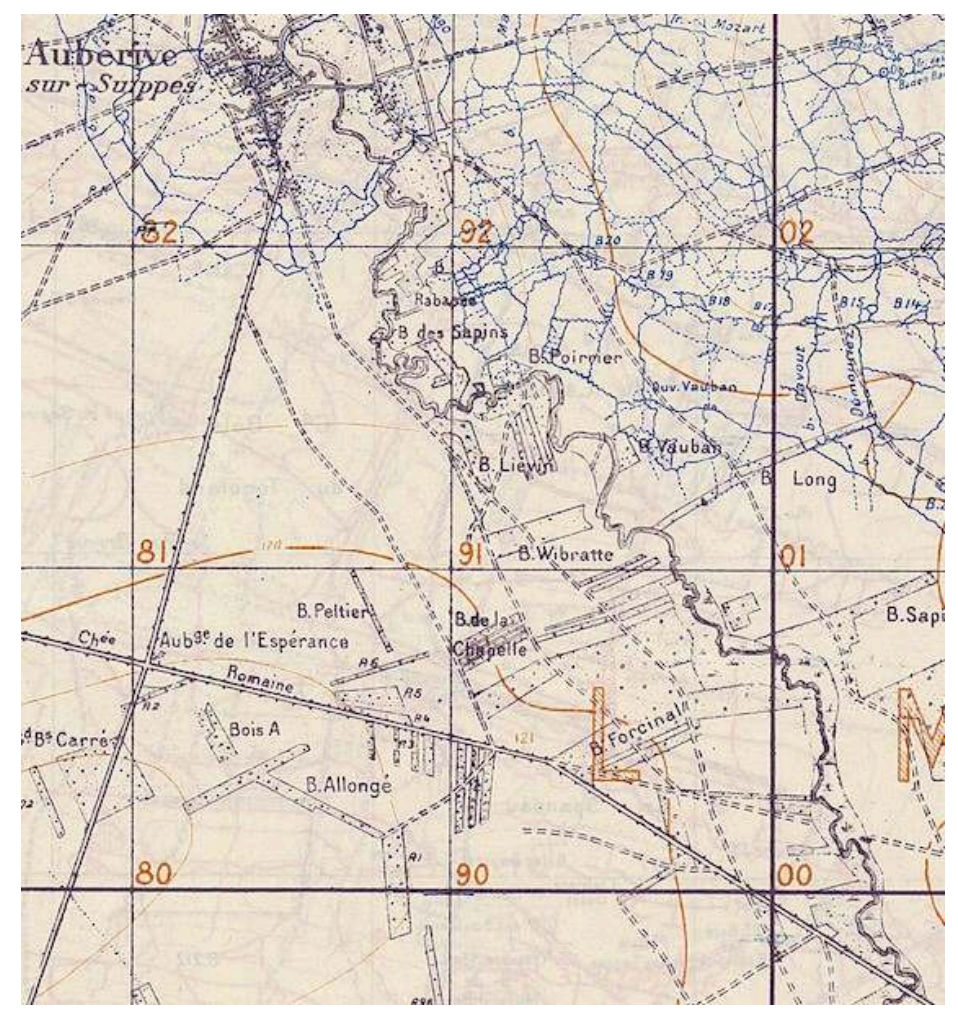

Document 3c : Repérage des bois pour replacer les points de découverte.

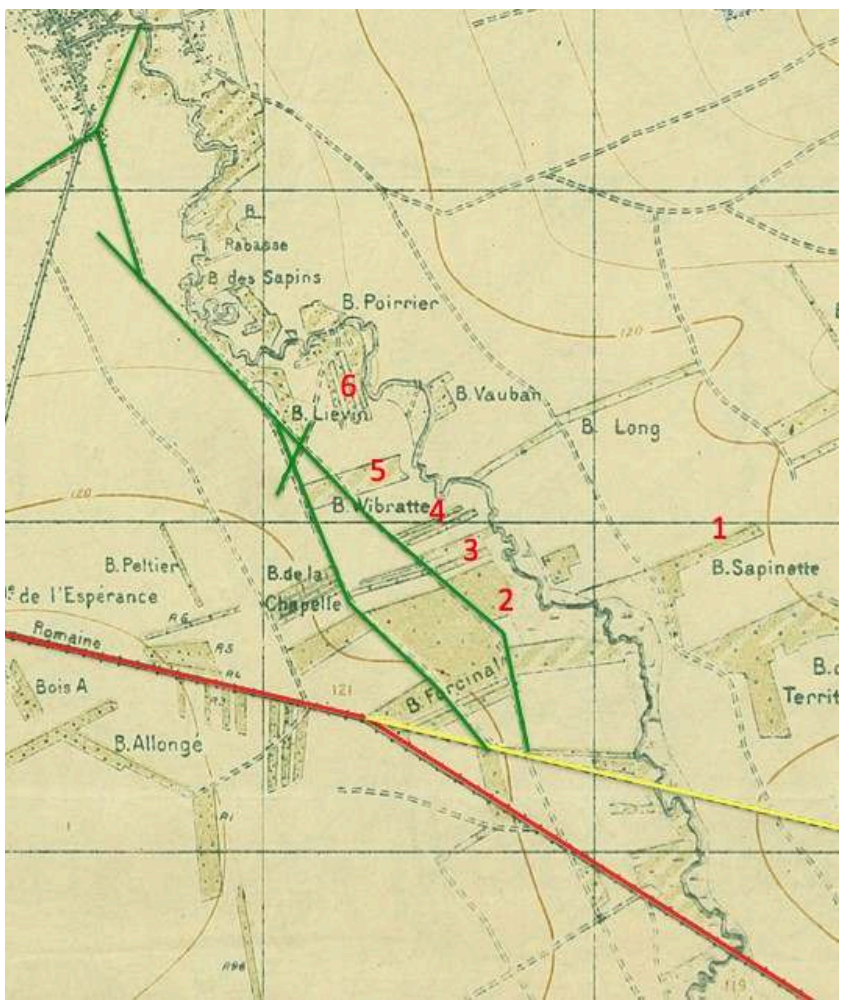


Document 4a : Localisation approximative des fosses explorées de part et d'autre de la limite communale d'Aubérive et de Saint-Hilaire-au-Temple (Marne).

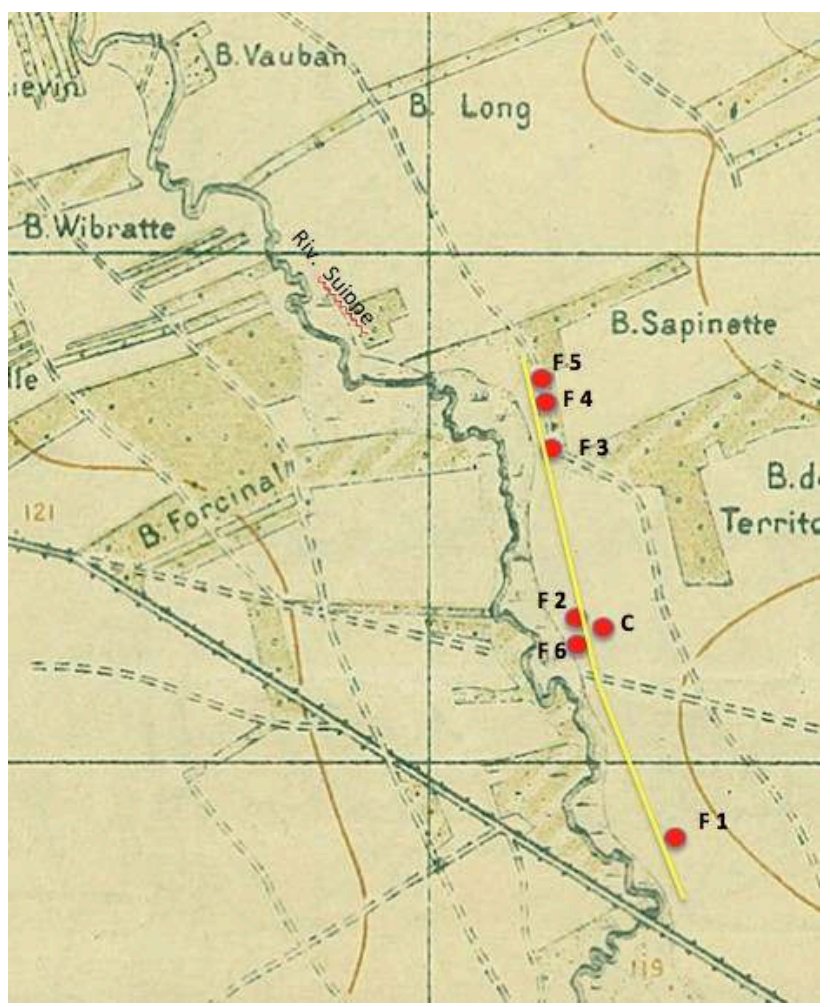

Document $4 b$ : Localisation approximative des découvertes sur Aubérive.

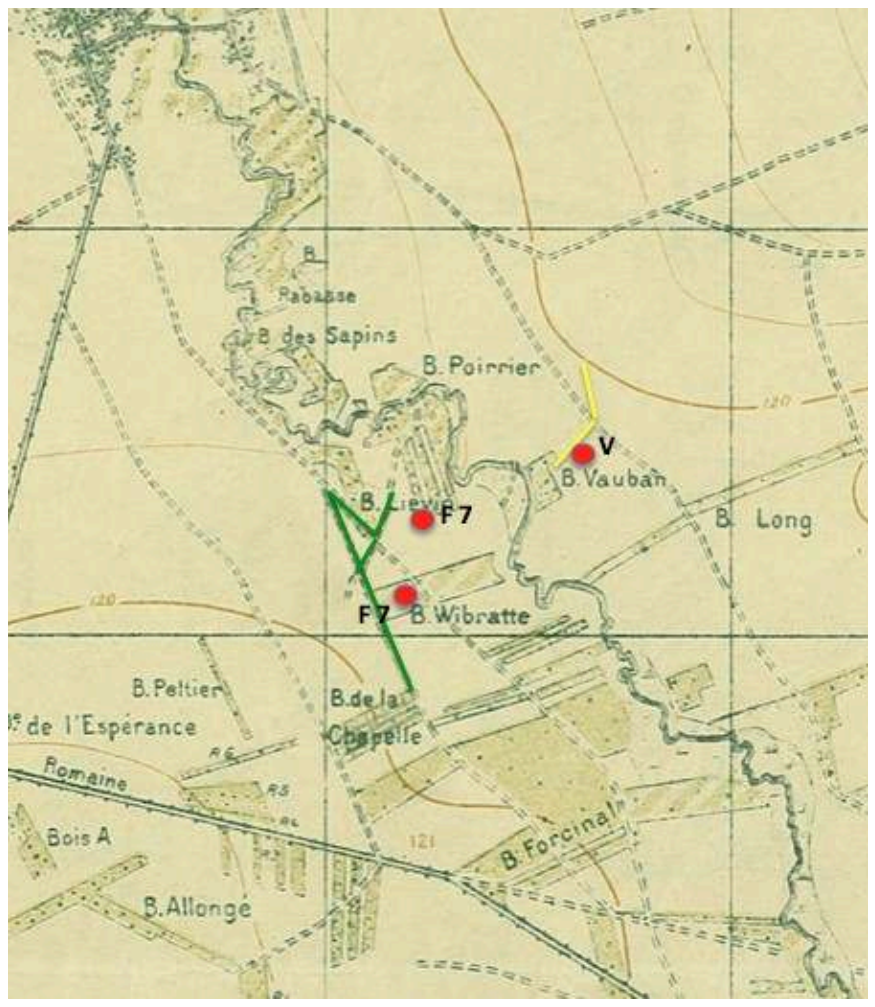

Le chiffre 7 peut être lu deux fois sur la carte originale de Trassagnac d'où la double position. C'est le point « $\mathrm{V}$ » correspondant au bois Vauban qui permet de situer avec le plus de précisions possible les découvertes de 1915. 
D'autres découvertes ont été signalées. Il est souvent évoqué l'épisode de la trouvaille d'une hache polie dans sa gaine dans une tranchée du Nord de la France (Barbusse, rééd. 1945). Mais, on peut ainsi relater les recherches inédites mentionnées dans les lettres et le carnet de route du Capitaine Léon Bérard dont on extrait les passages qui vont suivre (Bérard, 1920). La première mention remonte au 10 septembre 1914 : « [...] trouvé hier quelques beaux silex près de Rosières [...] (Aisne). C'est ensuite à la date du 14 septembre 1915, alors qu'il est en formation près de Châlons-sur-Marne puis à Sézanne qu'il note: "[...]j'ai passé le commencement de l'après-midi de la façon la plus inattendue, à chercher sans grand succès, des silex avec l'abbé Favret, Thiérot et Beuve le bibliothécaire [de Châlons] (sur le plateau du château de Frécul) [à Verdey près de Sézanne, Marne]. ». Puis c'est entre le 26 et le 31 juillet de la même année qu'il note : « En faisant préparer des tranchées pour un exercice de lancements de grenades, j'ai eu quelques instants pour chercher des silex et j'en ai encore trouvé quelques-uns, dont un ravissant petit grattoir ». Enfin le 9 février 1916, alors qu'il est cantonné dans les tranchées près de Prosnes (Marne), on peut lire: " [...] j'avais trouvé tout près de la voie romaine, une tombe qui semble intacte, mais je n'ai pas eu le temps de gratter. Elle est sûrement ancienne, mais l'est-elle assez pour contenir quelque chose de plus que les os? Je le verrai dans 6 jours. ». Le carnet reste malheureusement muet sur ce point. Par contre, le 20 du même mois, il écrit : «[...] si le temps est aussi beau, je m'accorderai trois heures de liberté, pour aller fouiller un vieux type, qui doit être un mérovingien, et que j'ai découvert en bordure d'un boyau, grâce à une dent qui dépassait! Quel triomphe, si je pouvais montrer à Thiérot quelque chose de bien! ».

On pense qu'il n'a pas eu le temps d'aller fouiller cette sépulture puisqu'il est envoyé dans les jours qui suivirent sur le front de Lorraine. Cette découverte est peut-être à rapprocher du site exploré par le fils Lelaurain en mai 1872 (Anonyme, 1872). Malgré la guerre, le trio d'archéologues, si actif de 1908 à juin 1914, se reforme pour se livrer à sa passion, pendant un court épisode de la guerre, celui de la formation de capitaine de Bérard chez les dragons alors qu'il était au $15^{\circ}$ chasseur à cheval. Favret (1875-1950) était aumônier à l'hôpital de Châlons et Thiérot (1881-1939) peut-être mobilisé sur Châlons mais libéré en 1919 à Strasbourg ? On ignore ce qu'est devenu le fruit des collectes de Bérard tout comme d'autres. Toutefois des objets réapparaissent de temps à autre mais, bien souvent sans origine précise, comme en atteste la plaque-boucle mérovingienne (legs Fontaine) mise au jour en 1914 pendant la bataille de la Marne (Anonyme, 2005).

En ce qui concerne la recherche allemande sur le front, le lecteur se reportera à plusieurs articles parus récemment pour l'Alsace et la Lorraine (Landolt et al., 2014; Landolt et al., 2017), pour le Nord et la Picardie (Desenne et al., 2009; Leman, 2017) et pour la Champagne concernée par le présent travail (Charpy, 2017). Lors du début de la guerre ce sont surtout des pillages qui ont été effectués. C'est seulement avec la guerre de positions et la mise en place du Kunstschutzen 1916 qu'à contrario des lignes françaises, côté allemand la recherche archéologique se développe. La fouille la plus remarquable a eu lieu sur les marges occidentales de la Champagne. C'est celle de trente-deux sépultures et d'une fosse entre le 8 février et le 9 avril 1915 par le soldat Hans Niggemann (1893-1981) et dont le mobilier est conservé au musée de Berlin. A la lumière des propos tenus par Trassagnac et rapportés plus haut, on perçoit mieux l'exploit réalisé par ce soldat allemand à Bucy-le-Long (Aisne) qui lui aussi était sur les premières lignes. Pour la Marne, c'est le site d'Aubérive (Chossenot, 2004; Charpy, 2017) qui est attesté par des archives du docteur Wolf (? - ?), notamment par trois 
lettres. La première est datée du 22 février 1916 et les deux autres des 5 et 11 mars 1916 mais les conditions de la fouille y sont assez peu explicites. La découverte concerne des tombes d'époque mérovingienne creusées dans une zone sableuse. D'autres recherches ont été pratiquées loin vers l'arrière, comme celles d'Heinrich Forschner (1880-1959), qui, en 1917-1918 se trouvait à Carignan dans les Ardennes (Basset et Vanmoerkerke, 2006 ; Vanmoerkerke, 2007) affecté à la $17^{\circ}$ compagnie du $76^{\mathrm{e}}$ R.I., et a exploré une villa gallo-romaine sur les pentes du Mont Tilleul. Il est allé jusqu'à acheter localement des collections pour les revendre plus tard au musée de Biberach (Allemagne). Toutefois, dans la Marne et les Ardennes, les recherches allemandes signalées sont bien moins nombreuses et moins riches qu'en Lorraine.

La géologie est restée jusqu'au premier Conflit mondial une science annexe de l'archéologie. Elle n'a eu d'intérêt pour les pionniers que de pouvoir donner de manière très lapidaire des informations sur l'environnement immédiat de leurs découvertes dont l'intérêt a surtout été celui de constituer des collections, dans un premier temps celles d'érudits (V. Duquenelle, A. Nicaise, L. Morel, J. de Baye, C. Bosteaux-Paris, etc.) mais aussi, d'un autre côté, de fouilleurs plus soucieux du rapport financier procuré par les objets (B. Lelaurain, P. Machet, etc.). L'intérêt géologique s'est porté sur certains types de pièces comme les objets en roches dures. C'est d'une part la rareté et d'autre part l'esthétique qui étaient les principaux moteurs pour évoquer ces matériaux. Lointaines sont les considérations sur l'origine, les déplacements de la matière première. Plus grande a été l'attention portée à l'identification des fossiles entrant dans la composition des éléments de la parure. Mais là encore a primé le rapport à la zoologie sur l'identification potentielle des provenances. A leur décharge, on argumentera sur la richesse des sables du Tertiaire et de leur proximité avec les lieux de découverte (Mont de Berru par exemple).

Les archéologues, comme les autres, ont été mobilisés et ont payé un lourd tribut (Déchelette en 1914, Bérard en 1918). Pour pouvoir exercer sur le terrain leur passion, il aura fallu des conditions bien particulières: le désœuvrement du casernement et l'accalmie sur le front. Mais à tout moment l'œil a cherché l'anomalie dans une coupe de tranchée, dans des travaux de terrassement. On note que côté français, les recherches ont été sans aucun doute plus nombreuses qu'on pouvait le penser mais, force est de constater que celles-ci ont été faites par des hommes qui avaient en eux cette passion déjà bien avant la guerre. Au travers des exemples recensés, on a pu mettre en évidence que selon leur intérêt, ils ont transmis des informations aux revues auxquelles eux même ou un de leurs proches étaient abonnés; ce qui explique les mentions dans des publications régionales. Les informations sont assez précises malgré la censure qu'ils s'imposent face à l'ennemi. Les objets ont connu des sorts différents, les uns perdus, les autres entrés dans les musées de villes (Toulouse), nationaux (MAN), de Société (S.P.F.). Le conflit a débuté par de nombreux pillages et de nombreuses destructions causés par l'armée allemande. Par la suite, elles ont été équivalentes face au feu des deux côtés mais la stabilité du front a amené l'administration germanique à créer un service de protection des œuvres d'art au sein de l'armée suite aux exactions du début de la guerre, et d'un service de recherches archéologiques en 1916. 


\section{BIBLIOGRAPHIE}

Anonyme, 1872, « Prosnes. », Le Journal de la Marne, n 7847, p. 2.

Anonyme, 1915a, « La grotte néolithique de Courjeonnet (Marne) », Bulletin de la Société préhistorique française, t. XII, fasc. 2, p. 71.

Anonyme, 1915b, « En creusant des tranchées », Le Temps, p. 3.

Anonyme, 2005, « L'activité scientifique du musée en 2005 », Antiquités nationales, n 37, pp. 12-13.

Barbusse H., 1945 (rééd.), Le feu, journal d’une escouade, Paris, Flammarion, (1 $1^{\text {ère }}$ ed. 1916), pp. 16-17.

Basset G. et Vanmoerkerke J., 2006, « Images de l'archéologie rurale en Champagne-

Ardenne », Bulletin de la Société archéologique champenoise, t. 99, p. 25.

Baye Baron J. de, 1880, L'archéologie préhistorique, Paris, Leroux, 411 p.

Baye Baron J. de, 1881, L'industrie acheuléenne dans le lœess de la Brie-Champenoise, Châlons-surMarne, Imp. Martin, 28 p.

Baye Baron J. de, 1886, « Un rapport archéologique entre l'ancien et le nouveau continent ", Matériaux pour l'Histoire primitive de l'Homme, $3^{\text {e}}$ série, t. III, octobre, pp. 477-481.

Beaudouin M., 1915, « La préhistoire dans les tranchées », Bulletin de la Société préhistorique française, t. XII, p. 275.

Bérard L., 1920, Lettres et carnet de route de Léon Bérard 1914-1918, Toulouse, Imp. du Sud-Ouest.

Bleicher M., 1880, « Application de la géologie microscopique à l'archéologie

préhistorique ", Association française pour l'Avancement des Sciences, $9^{\mathrm{e}}$ session, Reims, pp. 592-594.

Blog d'RV, 2005, « Liste des officiers du 216 R.I. entre 1914 et

1918 », neufneuf.canalblog.com,consulté en 2019.

Bosteaux-Paris C., 1883, « Cernay-lès-Reims. Sépultures gauloisesmarniennes de la nécropole des Barmonts ", Association française pour l'Avancement des sciences, congrès de Rouen, vol. II, pp. 586-592.

Bretz-Mahler D., 1971, « La civilisation de La Tène I en Champagne, le faciès marnien. », Gallia, XXIII' ${ }^{\mathrm{e}}$ suppl., Paris, CNRS, pp. 177-178.

Charpy J.-J., 2010, « L'archéologie régionale champenoise, à l'époque où Jean-Baptiste Counhaye a débuté ses recherches jusqu'à celle où il a donné sa collection à la Ville de Suippes » In : Lambot

B., Verger S., Cabart H., Charpy J.-J., Poulain C., « Jean-Baptiste Counhaye, sa collection à la mairie de Suippes (Marne) et l'archéologie champenoise au XIX ${ }^{\mathrm{e}}$ siècle. ", Mémoire de la Sociétéarchéologique champenoise, $\mathrm{n}^{\circ} 18, \mathrm{pp}$. 21-36.

Charpy J.-J., 2017, « L'archéologie champenoise dans le contexte du premier Conflit Mondial »In : Nivet P., LewuillonS., La Grande Guerre des archéologues, Actes du colloque d'Amiens 2016, Archéologues et historiens de l'art dans la Grande Guerre ,Dijon, Éditions universitaires de Dijon, coll. Histoires, pp. 187-202.

Chew H., 1993, « Une sépulture militaire de l'époque romaine tardive à Sarry (Marne) »In :Vallet F. et Kasanski M. ,L'armée romaine et les Barbares du III ${ }^{e} a u$ VII ${ }^{e}$ siècle, Rouen,Association française d'archéologie mérovingienne- Saint-Germain-en-Laye, Musée des Antiquités nationales, pp. 313-321. 
Chossenot R., 2004, «La Marne 51/1. ».Carte archéologique de la Gaule, Gap, Académie des Inscriptions et Belles-Lettres, pp. 179-180.

Crid1418, 2011, « Bonneau, Georges (1879-1969) », ～www.crid1418.org/temoins/2011/11/18/ bonneau-georges-1879-1969/ Consulté en 2019

Desenne S., Pommepuy C., Demoule J.- P., 2009, « Une nécropole de La Tène ancienne (V $\mathrm{IV}^{\mathrm{e}}$ siècles avant notre ère) ", Revue archéologique de Picardie, $\mathrm{n}^{\circ}$ spécial 26, vol. II, pp. 517-614. Goury G., 1911, L'enceinte d'Haulzy, Nancy, Les étapes de l'Humanité, vol. I, fasc. II, 107 p.

Huber M., 1915, «La préhistoire et les tranchées», Bulletin de la Société préhistorique française, t. 1, XII, fasc. 2, p. 68.

Jadart H., 1914, « Extrait de la lettre à Héron de Villefosse », Bulletin de la Société des Antiquaires de France, séance du 23 septembre 1914, p. 261, note 1.

Landolt M., Schnitzler B., Laparra, Mourot F., Legendre J.-P., 2014, « Des tranchées aux musées : l'archéologie pendant la Grande Guerre en Alsace et en Lorraine », In Situ. Revue des patrimoines, 23, [en ligne] URL : http://insitu.revues.org/10882.

Landolt M., Schnitzler B., Laparra, Mourot F., Legendre J.-P., 2017, « L'archéologie pendant la Grande Guerre en Alsace et en Lorraine. ».In : Nivet P. et LewuillonS.; La Grande Guerre des archéologues, Actes du colloque d'Amiens 2016, Archéologues et historiens de l'art dans la Grande Guerre, Dijon, Éditions universitaires de Dijon, coll. Histoires, pp. 259-307.

Laporte J.-P., 2017, « Gustave Hannezo (1857-1922) et l'archéologie tunisienne. » Cartagine, Studi e ricerche, 2, pp. 1-21.

Le Goffic C., 1916, « Les marais de Saint-Gond »,La Victoire de la Marne, Paris, Plon.

Le Goffic C., 1921, La Marne en feu, Paris, Alcan, (2 éd.).

Leman P., 2017, « Archéologues des Hauts-de-France de 1790 à nos jours », Archaïologia, Septentrion, $198 \mathrm{p}$.

Mougin Dr. L., 1877, « Fouille du cimetière gaulois de Charvais, territoire de HeiltzL’Evêque », Bulletin de la Société des arts et sciences de Vitry-le-François, t. VIII, pp. 245-268.

Mougin Dr. L., 1899, « Parure de Frignicourt, alluvions anciennes, âge des cavernes », Bulletin de la Société des arts et sciences de Vitry-le-François, t. XIX, 1896-1899, pp. 309-316.

Olivier L., Brière J., Multon H., 2015, « Le Musée des Antiquités nationales et la Grande Guerre », Antiquités nationales, 45, pp. 129-144.

Olivier Docteur P., 1916, « Les noms des tranchées ", L'intermédiaire des chercheurs et des curieux, vol. LXXIII, 20-29 février 1916, pp. 182-183.

Poinsignon M., 1877, Atlas de Géographie physique, administrative, historique et archéologique des 32 cantons de la Marne.

Polloni A., 2007, La parure dans les sépultures collectives de la fin du IVeau début du II millénaire en Bassin parisien.,Thèse de doctorat de l'Université Paris I,

Reinach S., 1917, « Une sépulture de La Tène I. », Bulletin archéologique du Comité des travaux historiques et scientifiques, pp. XIX et 74-75.

Reynier P., 1915, « La préhistoire des tranchées dans le canton de Lizy-sur-Ourcq (S.-et-M.). », Bulletin de la Société préhistorique française, t. XII, fasc. 5, pp. 245-246. 
Roland A., 1923, « Fouilles néolithiques de Villevenard, 8 mars 1915. », Bulletin de la Société archéologique champenoise, fasc. 2, pp. 45-46.

Trassagnac Docteur J., 1915a, « Fouilles dans les tranchées militaires. », Bulletin de la Société préhistorique française, t . XII, fasc. 5, p. 244.

Trassagnac Docteur J., 1915b, « Note sur quelques fouilles pratiquées dans les tranchées. », Bulletin de la Société préhistorique française, t. XII, fasc. 7 pp. 331-342.

Trassagnac Docteur J., 1916, « Dons à la S. P. F. », Bulletin de la Société préhistorique française, t. XIII, fasc. 5, pp. 243-244.

Trassagnac Docteur J., 1917a, « Découverte d'un puits funéraire gallo-romain à Tours-sur-Marne. ", Bulletin de la Société préhistorique française, t. XIV, fasc. 7, p. 348.

Trassagnac Docteur J., 1917b, « Le camp dit « Fossé des Sarrazins » de Mareuil-le-Port (Marne). », Bulletin de la Société préhistorique française, t. XIV, fasc. 2, pp. 153-158.

Vanmoerkerke J., 2007, « Carignan (Ardennes), Heinrich Forschner et l'archéologie pendant la Grande Guerre en Champagne-Ardenne. », Résumés de communication de la Journée archéologique de Champagne-Ardenne du 1er décembre, Châlons-en-Champagne, pp. 33-35.

\section{RÉSUMÉS}

Les archéologues de la seconde moitié du XIX ${ }^{\mathrm{e}}$ siècle ont beaucoup exploré les exploitations en carrière mais n'ont rendu compte des notions géologiques environnementales de leurs découvertes que par des mentions accompagnées d'un vocabulaire approprié dont la teneur reste très généraliste et sommaire. Les attributions sont en revanche très précises quand il s'agit d'identifier les restes fossiles de la faune marine du Crétacé supérieur ou des sables tertiaires du Lutétien, ou la nature des outils préhistoriques en roche étrangère à la région. Pendant le Premier Conflit mondial, les collections locales ont profondément souffert ou disparu. Les hypogées des marais de Saint-Gond, sauf un à Oyes, ont échappé à la destruction. Le considérable réseau de tranchées n'a pas permis la mise au jour ni le signalement de nombreuses découvertes (Aubérive/Saint-Hilaire-au-Temple, Cauroy-lès-Hermonville/Cormicy, Perthes-lès-Hurlus, Sarry). Les militaires, auteurs de rapports, ont tous un lien avec des sociétés savantes (Comité des travaux historiques, Société préhistorique française, Société archéologique du Midi de la France, etc.) et c'est vers elles que les objets trouvés ont été envoyés. Si l'armée allemande a vite mis en place un service, le Kunstschutz; il n'en est pas de même du côté des Alliés. Au sein du département de la Champagne-Ardenne, on retiendra, côté français, les travaux du médecin major J. Trassagnac à Aubérive/Saint-Hilaire-au-Temple, Tours-sur-Marne, Cerseuil (Marne) et, côté allemand, ceux de H. Forschner à Carignan (Ardennes).

Archaeologists from the second half of the nineteenth century have extensively explored quarry operations but have reported the environmental geological concepts of their discoveries only by mentions accompanied by an appropriate vocabulary whose content remains very general and summary. Attributions are however very precise when it comes to identifying the fossil remains of the marine fauna of the Upper Cretaceous or tertiary sands of the Lutetian, or the nature of the prehistoric stone tools foreign to the region. During the First World War, local collections suffered deeply or disappeared. Hypogea of the swamps of Saint-Gond, except one in Oyes, escaped destruction. The considerable network of trenches did not allow the discovery followed by a report of numerous discoveries (Aubérive / Saint-Hilaire-au-Temple, Cauroy-lèsHermonville / Cormicy, Perthes-lès-Hurlus, Sarry). The military authors of reports, all have a link 
with learned societies (Comité des travaux historiques, Société préhistorique française, Société archéologique du Midi de la France, etc.) and it is to them that the found objects were sent. If the German army quickly set up a dedicated department, the Kunstschutz ; it is not the same among allies. Within the department of Champagne-Ardenne, we notice, on the French side, the work of Major J. Trassagnac in Aubérive / Saint-Hilaire-au-Temple, Tours-sur-Marne, Cerseuil (Marne) and, on the German side, those of H. Forschner at Carignan (Ardennes).

\section{INDEX}

Keywords : WW1 Archaeology, Geoarchaeology, Trenches, Sedimentary model

Mots-clés : Archéologie de la Grande Guerre, Géoarchéologie, Tranchées, Modèle sédimentaire

\section{AUTEUR}

\section{JEAN-JACQUES CHARPY}

15, rue de l'Arquebuse, 51200 Epernay jj.charpy@wanadoo.fr 